\title{
Effect of surface treatment on molecular reorientation of polymer-stabilized liquid crystals doped with oligothiophene
}

\author{
Koji Usui ${ }^{1}$, Erika Katayama ${ }^{1}$, Jing Wang ${ }^{1}$, Kyohei Hisano ${ }^{1}$, Norihisa Akamatsu ${ }^{1}$ and Atsushi Shishido ${ }^{1,2}$ \\ Irradiation of dye-doped liquid crystals (LCs) with linearly polarized light leads to molecular reorientation, which manifests \\ functional properties for various nonlinear optical (NLO) applications. Material designs with lower light intensity thresholds for \\ molecular reorientation have been explored, and nematic LCs have been one of the most attractive choices because of the high \\ NLO properties. Here we present a different approach to reduce light intensity for reorientation by modifying a substrate surface \\ that controls initial molecular orientation in polymer-stabilized nematic LCs doped with oligothiophene. The surface of the \\ glass substrate was treated with various concentrations of a silane coupler. Water contact angle measurement and analysis of \\ samples using polarized optical microscopy revealed that surface anchoring in the initial state decreased as the silane coupler \\ concentration decreased. The threshold intensity was successfully reduced by $30 \%$ simply by optimizing the silane coupler \\ concentration. This finding clearly indicates that weak surface anchoring is key to the reduction of light intensity for molecular \\ reorientation.
}

Polymer Journal (2017) 49, 209-214; doi:10.1038/pj.2016.102; published online 19 October 2016

\section{INTRODUCTION}

Liquid crystal (LC) materials have had a great impact on our modern information society. ${ }^{1-3}$ Various LC materials and devices including LC displays, ${ }^{3}$ smart windows, ${ }^{4}$ reconfigurable optical elements ${ }^{5}$ and tunable optical metamaterials ${ }^{6}$ have been realized. ${ }^{2}$ In these applications, light modulation is the most important factor and is achieved by orientational changes of LC molecules using an external field.

Molecular reorientation of conventional LCs has been performed by an electric field. However, molecular reorientation triggered by an optical field has attracted attention because it enables the development of all-optical devices. ${ }^{7,8}$ Such photoinduced molecular reorientation includes photochemical and photophysical processes. Photochemical processes control LC orientation through a photochemical reaction, for example, photoisomerization, photocrosslinking or photodegradation. ${ }^{8-14}$ In contrast, a photophysical process exploits the nonlinear optical (NLO) effects of an LC.,15 When a homeotropic LC is vertically irradiated with linearly polarized light, the interaction between the optical field and the LC molecules generates a torque to rotate the molecular director along the polarization direction, leading to homogeneous (in-plane) orientation. On the other hand, rotation of the molecular director is disturbed both by the interaction between LCs and the glass substrate surface, a process which is also called surface anchoring, and by bulk elasticity. These torques reach a balance that determines a specific light intensity that allows reorientation of the molecular director, which means that the reorientation behavior has a well-defined threshold light intensity. ${ }^{16}$ This NLO effect on molecular reorientation has attracted attention owing to high NLO efficiency. ${ }^{17-22}$ When the initial homeotropic (out-of-plane) orientation is changed to homogeneous orientation with light, a large change in the refractive index is induced, which leads to a large NLO effect. In addition, nematic LCs that have high optical nonlinearity reorient the molecular director at lower light intensity compared with conventional materials. ${ }^{23}$ To date, applications such as data storage, ${ }^{24}$ efficient optical switching ${ }^{25}$ and light beam modulation ${ }^{26}$ have been reported. This process, however, still requires relatively higher-power polarized light sources compared with photochemical processes. Therefore, the most important issue is the reduction of the threshold intensity for molecular reorientation, which would expand photonic applications.

A series of studies succeeded in reducing the threshold intensity in homeotropic nematic LC systems. In 1990, Jánossy et al. ${ }^{15}$ first reported that the threshold light intensity for photoinduced molecular reorientation greatly decreases in an LC doped with a small amount of anthraquinone dye. ${ }^{27}$ In this system, the torque to rotate LCs is caused by an optical field and is enhanced by the molecular polarizability of photoexcited dyes. Thus, the threshold intensity decreases. In 2000, Zhang et al. ${ }^{28}$ reported that doping LCs with an oligothiophene derivative further reduced the threshold intensity. Later, Yaegashi et al.

\footnotetext{
${ }^{1}$ Laboratory for Chemistry and Life Science, Institute of Innovative Research, Tokyo Institute of Technology, Yokohama, Japan and ${ }^{2}$ Precursory Research for Embryonic Science and Technology (PRESTO), Japan Science and Technology Agency (JST), Kawaguchi, Japan

Correspondence: Professor A Shishido, Laboratory for Chemistry and Life Science, Institute of Innovative Research, Tokyo Institute of Technology, R1-12, 4259 Nagatsuta, Midoriku, Yokohama 226-8503, Japan.

E-mail: ashishid@res.titech.ac.jp

Received 30 June 2016; revised 12 September 2016; accepted 15 September 2016; published online 19 October 2016
} 
synthesized oligothiophene derivatives with ester moieties directly connected to the terminal thiophene to enhance polarizability of the oligothiophene. As a result, the threshold intensity was lowered 3-fold compared to LC doped with the original oligothiophene. ${ }^{29}$ Thus, reduction has been achieved but mainly through the development of more polarizable dyes.

Although the importance of surface anchoring and bulk elasticity has been well known theoretically, attempts to reduce the threshold intensity by controlling surface anchoring are rare, except for the command surface concept. ${ }^{30-34}$ Seki et al..$^{33}$ and Aoki et al. ${ }^{34}$ revealed that the packing density of azobenzene units over the substrate surface influences the molecular reorientation behavior of LCs. Here we report that surface treatment drastically affects and reduces the threshold intensity for molecular reorientation in dye-doped LCs. Two recent findings in our group suggested that threshold intensity is greatly dependent on surface anchoring. We reported that a homeotropic-aligned polymer-stabilized LC (PSLC) with a relatively low concentration of LC polymers enhances optical nonlinearity and allows the reduction of the threshold intensity by a factor of 6 compared with conventional homeotropic LCs. ${ }^{35}$ The reduction is attributed to the disturbance of surface anchoring by the polymers. In addition, we found that a hybrid-aligned PSLC, in which the LC is initially oriented in a homeotropic manner on one surface and in a homogeneous manner on the other, also decreases the threshold intensity by a factor of 8.5 compared with conventional homeotropicaligned LCs. ${ }^{36}$ This reduction in threshold intensity was explained by a decrease in total surface anchoring. When one surface was treated to show a homogeneous orientation, the necessary torque for photoinduced reorientation decreased. In the present report, we focused on a surface treatment using a silane coupler, which induces the initial homeotropic orientation of LCs. ${ }^{36,37}$ This study revealed that surface treatments affect the threshold intensity for photoinduced molecular reorientation in PSLCs. As the silane coupler concentration decreased, the threshold intensity was reduced by up to $30 \%$, suggesting a principle for decreasing the threshold intensity for molecular reorientation and paving the way for the further development of photonic devices.

\section{EXPERIMENTAL PROCEDURES}

\section{Materials}

The chemical structures of the compounds used in this study are shown in Figure 1. As a host nematic LC, 5CB (4-cyano-4'-pentyl biphenyl) was obtained from Merck Ltd., Tokyo, Japan, and used without further purification. The photopolymerizable monomer, A4CB (4-[4-(4'-cyanobiphenyl)oxy]butyl acrylate), was synthesized according to a procedure similar to that used in a previous report. ${ }^{38}$ The photoresponsive dye molecule, TR5 (5,5"-Bis-(5-butyl2-thienylethynyl)-2,2':5',2"-terthiophene), was synthesized as reported previously. ${ }^{28}$ Irgacure 651 was used without further purification as the photoinitiator. Surface modification was performed with a silane coupling agent (silane coupler), octadecyltrimethoxysilane, purchased from Tokyo Chemical Industry Co., Ltd., Tokyo, Japan.

\section{Surface modification of glass substrates}

The silane coupling treatment of glass substrates was carried out as follows. Glass substrates $\left(75 \times 25 \mathrm{~mm}^{2}\right)$ were ultrasonically cleaned with neutral detergent (Cica clean LX-II, Kanto Chemical Co., Inc., Tokyo, Japan), distilled water and 2-propanol. Subsequently, the substrates were treated with a ultraviolet (UV)-ozone cleaner. The cleaned substrates were immersed in an ethanol solution with the silane coupler at concentrations of $0,0.001,0.003$, $0.005,0.006,0.008,0.01,0.05$ or $0.15 \mathrm{wt} \%$ for $30 \mathrm{~min}$. Heating at $100{ }^{\circ} \mathrm{C}$ for $1.5 \mathrm{~h}$ yielded surface-treated glass substrates. a

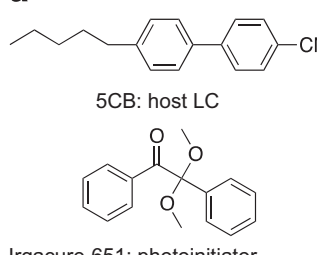

Irgacure 651: photoinitiator

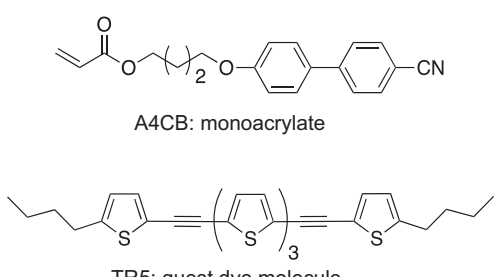

TR5: guest dye molecule b

$$
\begin{gathered}
-0 \\
-0.1 \\
\text { Si } \\
1 \\
\text { - }
\end{gathered}
$$

Octadecyltrimethoxysilane: silane coupling agent

Figure 1 (a, b) Chemical structures of compounds used in this study for preparation of dye-doped PSLCs and surface treatment.

Water contact angle measurements were carried out to investigate the surface properties of these substrates, the water contact angle was measured at five positions on every glass substrate and the average value was used as the net contact angle at each concentration.

\section{Preparation of LC cells}

Glass cells were handmade by bonding two surface-treated substrates with a 100- $\mu \mathrm{m}$-thick spacer. Schematic illustrations of the preparation of a glass cell are shown in Figure 2. Next, 5CB, the host nematic LC, and A4CB, an acrylate monomer, were mixed at a molar ratio of 90:10 in a brown vial, and Irgacure 651 was added to the mixture at a concentration of $0.5 \mathrm{~mol} \%$. A TR 5 solution $\left(0.01 \mathrm{~mol} \mathrm{l}^{-1}\right.$ in tetrahydrofuran) was doped into the mixture of $5 \mathrm{CB}$ and $\mathrm{A} 4 \mathrm{CB}$ at a dye concentration of $0.1 \mathrm{~mol} \%$. The obtained mixture was dissolved in THF and stirred for $1 \mathrm{~h}$. After the solvent was completely removed under vacuum, the mixture was injected into the glass cell by capillary action at room temperature. Photopolymerization was performed for $1 \mathrm{~h}$ by irradiation with 366-nm UV light $\left(1.0 \mathrm{~mW} \mathrm{~cm}^{-2}\right)$ from a high-pressure mercury lamp equipped with neutral density filters and band-pass filters (ND-70, UVD36A, UV-35, IRA-25S, AGC Techno Glass, Co., Ltd., Shizuoka, Japan). The cell was heated to $70{ }^{\circ} \mathrm{C}$ and gradually cooled to room temperature at $2{ }^{\circ} \mathrm{C}$ min ${ }^{-1}$. The photopolymerized LC cell was kept in the dark for $24 \mathrm{~h}$ to stabilize the initial molecular orientation of the obtained PSLCs.

\section{Polarized optical microscopy}

The initial molecular orientation in the PSLC cells was observed with a polarized optical microscope (BX50, Olympus, Tokyo, Japan) equipped with an interference filter at $550 \mathrm{~nm}$, where the sample has no absorbance.

\section{Ultraviolet-visible absorption spectroscopy}

A polarized ultraviolet-visible absorption spectrophotometer (V-650ST, JASCO Corporation, Tokyo, Japan) equipped with a polarizer was employed to confirm the dye orientation in the PSLC cell. Absorbance in the wavelength range of 350-600 $\mathrm{nm}$ due to TR5 was measured.

\section{Self-diffraction ring measurement}

We performed self-diffraction ring measurements to quantitatively evaluate any photoinduced molecular reorientation behavior. The optical setup used in the experiment is shown in Figure 3. The PSLC cell was irradiated with a vertically polarized $\mathrm{Ar}^{+}$laser beam (488 nm, BeamLok, 2060ZGB, Spectra Physics, Tokyo, Japan) transmitted with a Gran-Thompson prism, a convex lens (focal length: $15 \mathrm{~cm}$ ) and a variable neutral density filter. The shape of the incident and transmitted beams was measured with a beam profiler (BeamStar FX-50, Ophir Japan Ltd., Saitama, Japan).

\section{RESULTS AND DISCUSSION}

\section{Effect of surface modification by silane coupler}

The water contact angle of the glass surfaces treated with a silane coupler was measured (Figure 4). Although the water contact angle of 


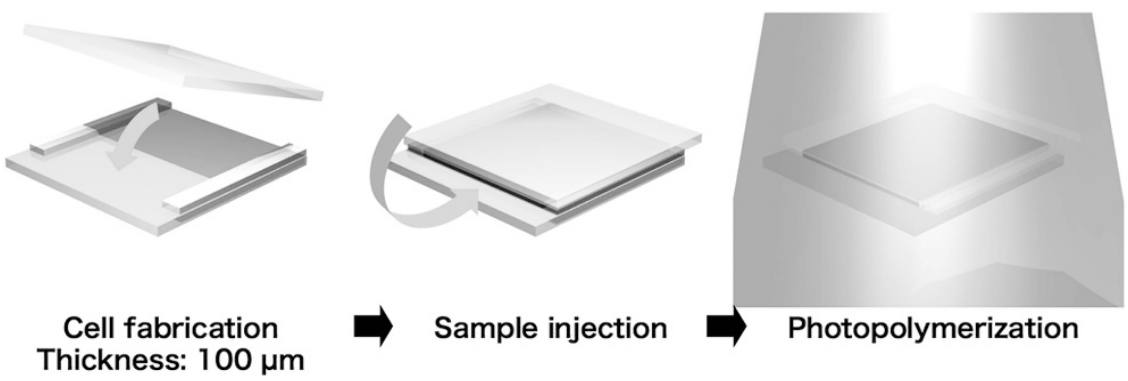

Figure 2 Schematic illustrations of the fabrication procedure for a homeotropic-aligned oligothiophene-doped PSLC cell. A full color version of this figure is available at Polymer Journal online.

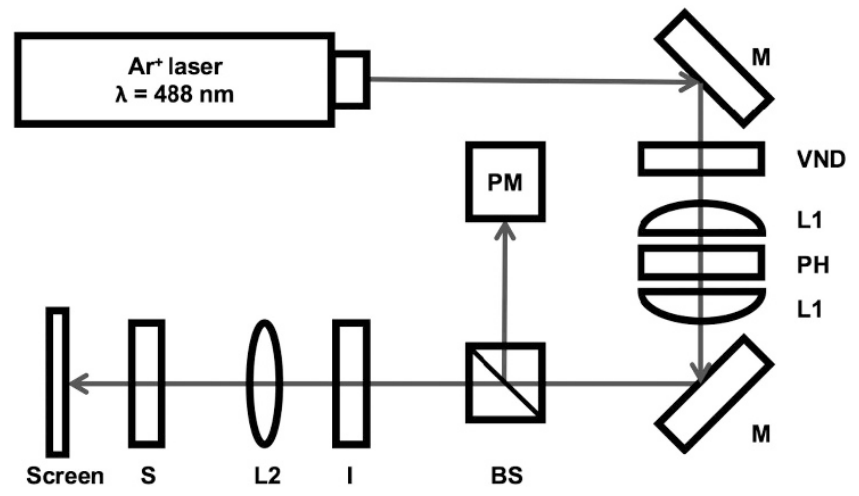

Figure 3 The incident laser beam was vertically polarized. BS, beam splitter; I, iris; L1, plane convex lens; L2, biconvex lens $(f=15 \mathrm{~cm})$; M, mirror; $\mathrm{PH}$, pinhole; PM, power meter; S, sample cell; VND, variable neutral density filter. A full color version of this figure is available at Polymer Journal online.

the glass surface after UV-ozone treatment was $\sim 0^{\circ}$, a glass substrate treated with pure ethanol without a silane coupler exhibited a water contact angle of $\sim 30^{\circ}$. Moreover, when a substrate was treated with an ethanol solution with a silane coupler at a concentration above $0.001 \mathrm{wt} \%$, the water contact angle drastically and monotonically increased. These results show that the water contact angle is related to surface anchoring and to the magnitude of the coverage of the silane coupler on the surface. The alkyl spacer of the silane coupler over the surface generates surface anchoring and induces the homeotropic orientation of LC molecules. ${ }^{36,37}$

\section{Characterization of LC orientation}

Polarized optical microscopy analysis of the PSLCs revealed that surface treatment by the silane coupler at concentrations above $0.003 \mathrm{wt} \%$ yielded a uniform and optically transparent yellow color (top photographs in Figure 5a and b). Conoscopic polarized optical microscope images of the cells exhibited clear isogyre (middle photographs in Figure 5a and b). This finding indicates that PSLCs have a homeotropic orientation in the cell. In addition, the homeotropic orientation of both host LCs and guest dyes was evident by polarized UV-visible absorption spectroscopy (bottom graphs in Figure $5 \mathrm{a}$ and $\mathrm{b}$ ). The vertical and horizontal absorption spectra in the wavelength range of the host LCs and the guest dyes were the same, independent of the polarization direction. In contrast, as shown in Figure $5 c$, the cell treated with a $0.001 \mathrm{wt} \%$ silane coupler solution exhibited a partially hazy appearance arising from microdomains. As shown in Figure $5 \mathrm{~d}$, the cells treated with $0 \mathrm{wt} \%$ silane coupler solution became opaque and never exhibited isogyre with a polarized

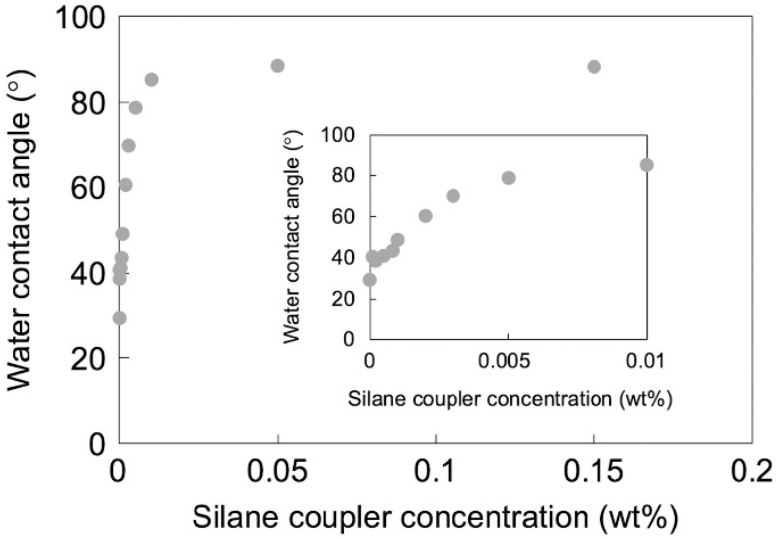

Figure 4 Water contact angle of glass substrates treated with a silane coupler as a function of silane coupler concentration. A full color version of this figure is available at Polymer Journal online.

optical microscope. In polarized UV-visible spectroscopy, the absorption spectra of the cell treated with a $0.001 \mathrm{wt} \%$ solution depended on the polarization direction of the probe light. Moreover, the absorbance of the cell treated with a $0 \mathrm{wt} \%$ solution showed clear polarization dependence (bottom graphs in Figure $5 \mathrm{c}$ and d). Therefore, PSLC cells treated with a sufficient amount of a silane coupler are induced to a uniform homeotropic orientation. At silane coupler concentrations below $0.001 \mathrm{wt} \%$, surface anchoring is not sufficient to maintain a uniform homeotropic orientation over a glass substrate, as indirectly verified by the water contact angle measurements. To quantitatively discuss the effect of silane couplers on anchoring, further experiments, such as those as reported by Aoki et al., ${ }^{34}$ are needed.

\section{Evaluation of optical nonlinearity of PSLCs}

Based on the NLO effect of the LCs, the photoinduced orientational change of LC molecules exhibits a clear dependence on light intensity, as described above. The threshold intensity is determined by three balancing factors that interact with an optical field of polarized light: molecular polarizability, bulk elasticity and surface anchoring. Considering previous reports, ${ }^{16,30,31}$ it is assumed that the threshold intensity for photoinduced molecular reorientation can be reduced by weakening the surface anchoring. This assumption was confirmed by direct evaluation of optical nonlinearity in self-diffraction ring measurements. ${ }^{15,16,39}$ The following experiments were carried out on PSLC cells treated with silane coupler concentrations above $0.003 \mathrm{wt} \%$ because these concentrations yielded a uniform homeotropic orientation. 
a
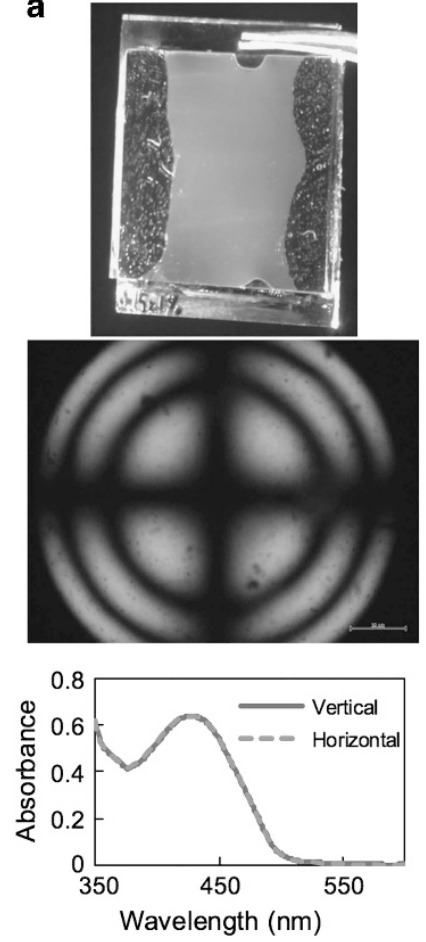

b
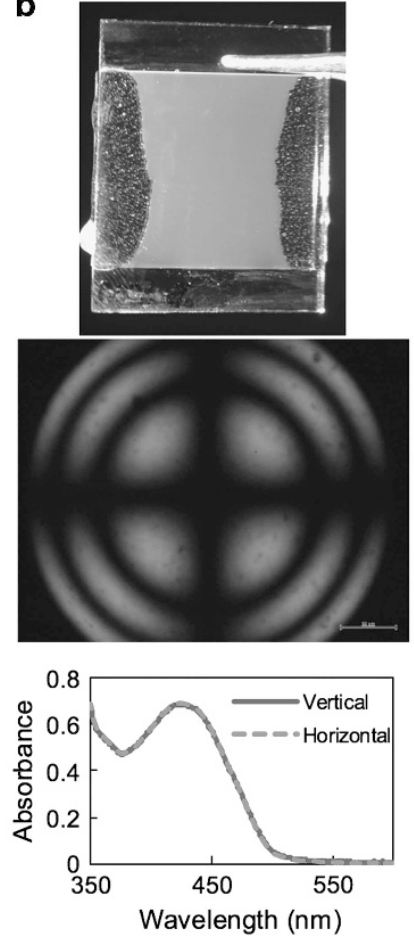

c
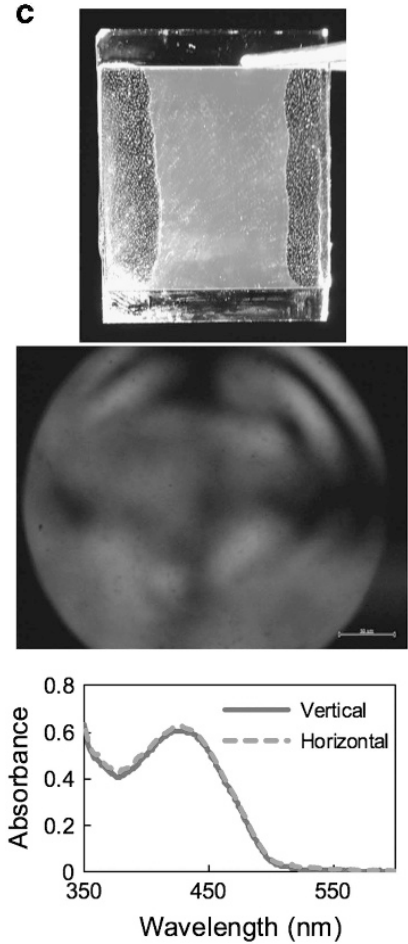

d
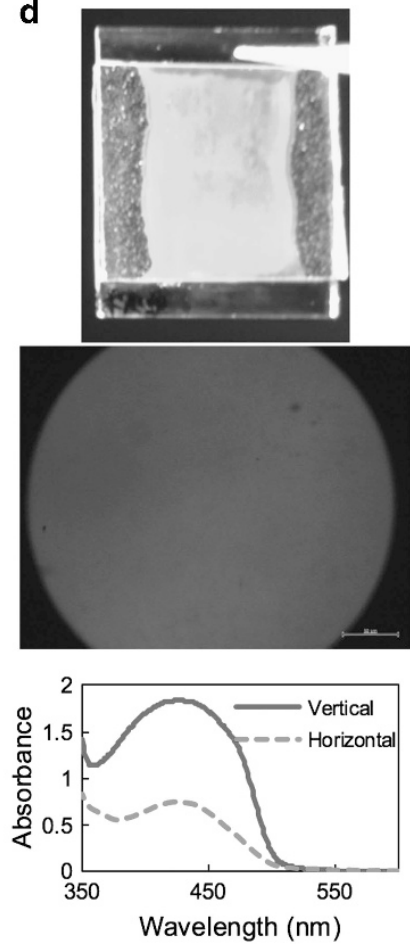

Figure 5 Photographs (top), conoscopic polarized optical microscope images (middle) and polarized UV-vis absorption spectra (bottom) of PSLCs treated with a silane coupler at the concentrations of 0.15 (a), 0.003 (b), 0.001 (c) and 0 wt\% (d). A full color version of this figure is available at Polymer Journal online.

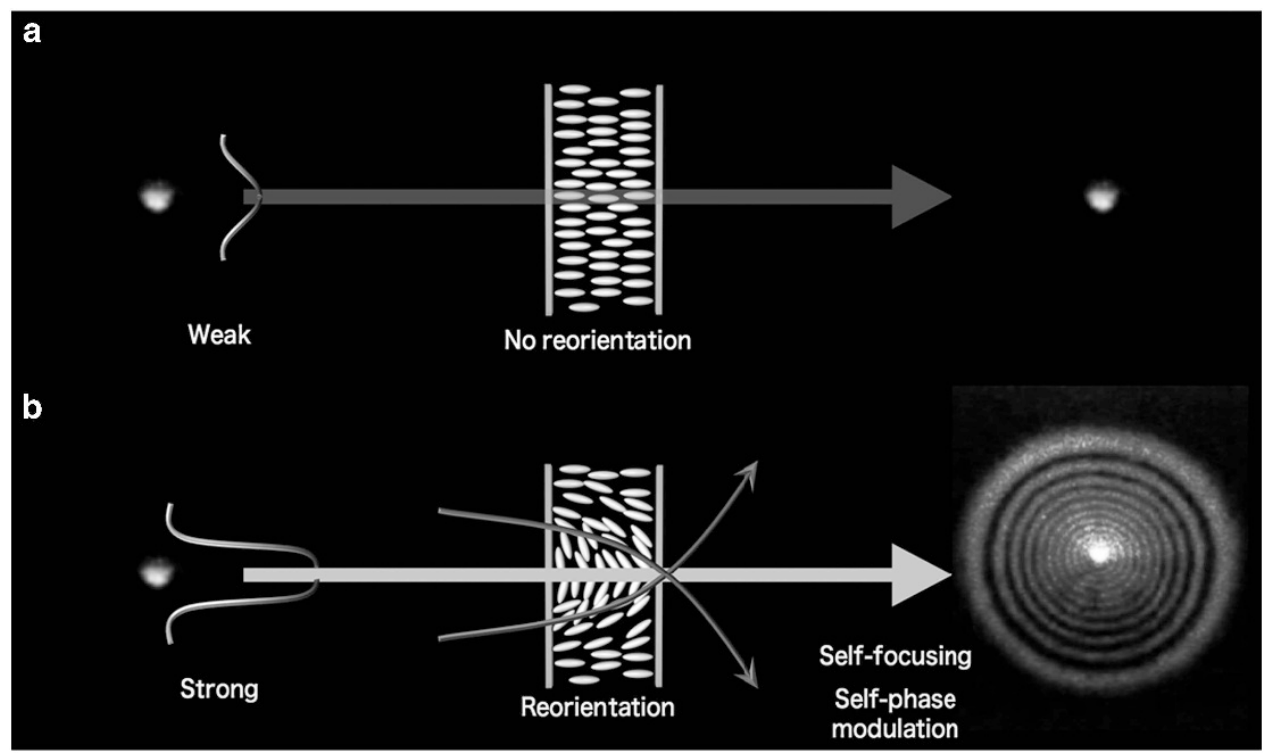

Figure 6 Principle of self-diffraction ring measurement. (a) Molecular reorientation is observed when an incident laser beam is weak. (b) Above the threshold intensity, molecular reorientation is induced, accompanied by the formation of a diffraction ring pattern through self-focusing and self-phase modulation. A full color version of this figure is available at Polymer Journal online.

When cells were irradiated with a linearly polarized $\mathrm{Ar}^{+}$laser beam, diffraction rings were observed on the screen above a certain light intensity, as shown in Figure 6. The number of rings increased as light intensity increased, and in all cells, the maximum number of rings was 27. Irradiation of the PSLCs with a low-intensity Gaussian beam did not cause molecular reorientation (Figure 6a). This means that when light intensity of an incident laser beam increases and exceeds the threshold, reorientation occurs, accompanied by the formation of selfdiffraction rings on a screen, as shown in Figure 6b. The generation of these rings arises from self-focusing and self-phase modulation owing 


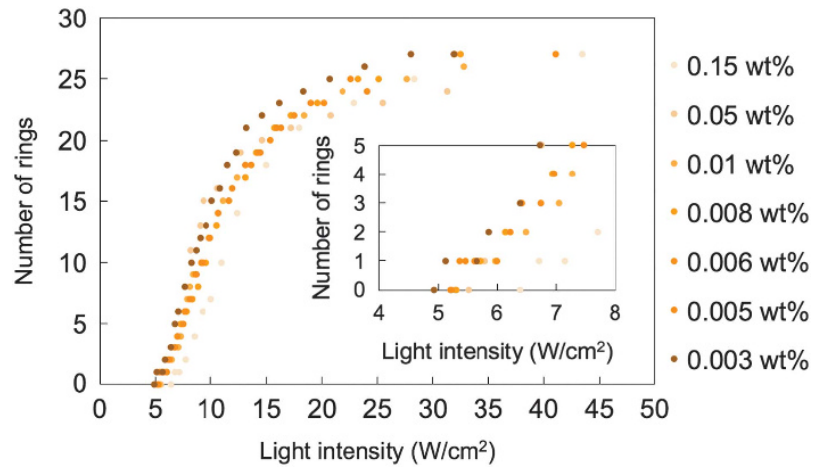

Figure 7 Number of diffraction rings as a function of light intensity for dyedoped PSLC cells treated with various concentrations of a silane coupler. The threshold intensities were $6.4 \mathrm{~W} \mathrm{~cm}^{-2}$ for $0.15 \mathrm{wt} \%, 5.5 \mathrm{~W} \mathrm{~cm}^{-2}$ for $0.05 \mathrm{wt} \%, \quad 5.3 \mathrm{~W} \mathrm{~cm}^{-2}$ for $0.01 \mathrm{wt} \%, \quad 5.28 \mathrm{~W} \mathrm{~cm}^{-2}$ for $0.008 \mathrm{wt} \%$, $5.21 \mathrm{~W} \mathrm{~cm}^{-2}$ for $0.006 \mathrm{wt} \%, 5.21 \mathrm{~W} \mathrm{~cm}^{-2}$ for $0.005 \mathrm{wt} \%$ and $4.9 \mathrm{~W} \mathrm{~cm}^{-2}$ for $0.003 w t \%$.

to molecular reorientation. Therefore, the molecular reorientation behavior can be evaluated quantitatively by counting the number of rings $(N)$ associated with the photoinduced refractive index change $\Delta n^{\prime}$ using the following equation, $N=\left|\Delta n^{\prime}\right| L \lambda^{-1}$ ( $\lambda$ : wavelength, $488 \mathrm{~nm}$; $L$ : cell thickness, $100 \mu \mathrm{m}){ }^{39}$ The value of $\Delta n^{\prime}$ was estimated to be 0.13 . It is noteworthy that molecules are reoriented almost parallel to the polarization direction of the incident beam by assuming that the birefringence of the PSLCs is equivalent to that of the host liquid crystal, 5CB. This finding clearly indicates that the present system can induce sufficient refractive index changes regardless of silane coupler concentrations.

Self-diffraction rings were observed to determine the threshold intensity, which we defined as the light intensity at which the first diffraction ring appeared on the screen. As shown in Figure 7, among all cells, the cells treated with a $0.003 \mathrm{wt} \%$ silane coupler solution showed the lowest threshold intensity $\left(4.9 \mathrm{~W} \mathrm{~cm}^{-2}\right)$, which was $30 \%$ lower compared with that treated with a $0.15 \mathrm{wt} \%$ solution. The threshold intensity increased with the concentration of the silane coupler, which can increase surface anchoring. Consequently, we suggest that a reduced threshold intensity is caused by a reduction in surface anchoring that controls the initial alignment of PSLC molecules.

\section{CONCLUSION}

We greatly reduced the threshold intensity for inducing molecular reorientation of PSLCs by controlling the surface treatment of glass substrates. The optical nonlinearity owing to molecular reorientation was quantitatively evaluated using measurement of self-diffraction rings arising from self-focusing and self-phase modulation effects. The threshold intensity decreased as surface anchoring was weakened. For the cells treated with a $0.003 \mathrm{wt} \%$ silane coupler solution, the threshold intensity was reduced by $30 \%$ compared with the highest silane coupler concentration. We believe that further decreases in the threshold intensity of NLO in dye-doped LCs or PSLCs will lead to the development of photonic materials and devices, such as a novel material that shields only high-intensity light.

\section{CONFLICT OF INTEREST}

The authors declare no conflict of interest.

\section{ACKNOWLEDGEMENTS}

This work was supported by the Precursory Research for Embryonic Science and Technology (PRESTO), Molecular Technology and Creation of New Functions, Japan Science and Technology Agency (JST).

1 Kato, T., Mizoshita, N. \& Kishimoto, K. Functional liquid-crystalline assemblies selforganized soft materials. Angew. Chem. Int. Ed. 45, 38-68 (2006).

2 Khoo, I. C. Nonlinear optics of liquid crystalline materials. Phys. Rep. 471, 221-267 (2009).

3 Schadt, M. Liquid crystal materials and liquid crystal displays. Annu. Rev. Mater. Sci. 27, 305-379 (1997).

4 Lampert, C. M. Large-area smart glass and integrated photovoltaics. Sol. Energy Mater. Sol. Cells 76, 489-499 (2003).

5 Tabiryan, N. V., Nersisyan, S. R., Steeves, D. M. \& Kimball, B. R. The promise of diffractive waveplates. Opt. Photon. News 21, 40-45 (2010).

6 Khoo, I. C., Diaz, A., Liou, J., Stinger, M. V., Huang, J. \& Ma, Y. Liquid crystals tunable optical metamaterials. IEEE J. Sel. Topics Quantum Electron. 16, 410-417 (2010).

7 Tabiryan, N. V., Sukhov, A. V. \& Zel'dovich, B. Y. The orientational optical nonlinearity of liquid crystals. Mol. Cryst. Liq. Cryst. 136, 1-139 (1986).

8 Seki, T. New strategies and implication for the photoalignment of liquid crystalline polymers. Polym. J. 46, 751-768 (2014).

9 Priimagi, A., Barrett, C. \& Shishido, A. Recent twists in photoactuation and photoalignment control. J. Mater. Chem. C 2, 7155-7162 (2014).

10 Fukuhara, K., Nagano, S., Hara, M. \& Seki, T. Free-surface molecular command systems for photoalignment of liquid crystalline materials. Nat. Commun. 5 3320 (2014).

11 Ichimura, K., Suzuki, Y., Seki, T., Hosoki, A. \& Aoki, K. Reversible change in alignment mode of nematic liquid crystals regulated photochemically by command surfaces modified with an azobenzene monolayer. Langmuir 4, 1214-1216 (1988).

12 Schadt, M., Schmitt, K., Kozinkov, V. \& Chigrinov, V. Surface-induced parallel alignment of liquid crystals by linearly polymerized photopolymers. Jpn. J. Appl. Phys. 31, 2155-2164 (1992).

13 Minami, S., Kondo, M. \& Kawatsuki, N. Fabrication of UV-inactive photoaligned films by photoinduced orientation of $\mathrm{H}$-bonded composites of non-photoreactive polymer and cinnamate derivative. Polym. J. 48, 267-271 (2016).

14 Hasegawa, M. \& Taira, Y. Nematic homogeneous photo alignment by polyimide exposure to linearly polarized UV. J. Photopolym. Sci. Technol. 8, 241-248 (1995).

15 Jánossy, I., Lloyd, A. D. \& Wherrett, B. S. Anomalous optical Freedericksz transition in an absorbing liquid crystal. Mol. Cryst. Liq. Cryst. 179, 1-12 (1990).

16 Marrucci, L. Mechanisms of giant optical nonlinearity in light-absorbing liquid crystals: a brief primer. Liq. Cryst. Today 11, 1-28 (2002).

17 Zolot'ko, A. S., Kitaeva, V. F., Kroo, N., Sobolev, N. N. \& Chillag, L. The effect of an optical field on the nematic phase of the liquid crystal OCBP. JETP Lett. 32, 158-162 (1980).

18 Zel'dovich, B. Y., Pilipetskii, N. F., Sukhov, A. V. \& Tabiryan, N. V. Giant optical nonlinearity in the mesophase of a nematic liquid crystal (NCL). JETP Lett. 31, 263-269 (1980).

19 Durbin, S. D., Arakelian, S. M. \& Shen, Y. R. Optical-field-induced birefringence and Freedericksz transition in a nematic liquid crystal. Phys. Rev. Lett. 47, 1411-1414 (1981).

20 Eich, M., Wendorff, J. H., Ringsdorf, H. \& Schmidt, H. W. Nonlinear optical self diffraction in a mesogenic side chain polymer. Macromol. Chem. Phys. 186, 2639-2647 (1985).

21 Shtykov, N. M., Barnik, M. I., Beresnev, L. A. \& Blinov, L. M. A study of a ferroelectric liquid crystal using second optical Harmonic generation. Mol. Cryst. Liq. Cryst. 124, 379-390 (1985).

$22 \mathrm{Khoo}, \mathrm{I}$. C. Optically induced molecular reorientation and third-order nonlinear optical processes in nematic liquid crystals. Phys. Rev. A 23, 2077-2081 (1981).

23 Moran, M. J., She, C.-Y. \& Carman, R. L. Interferometric measurements of the nonlinear refractive-index coefficient relative to $\mathrm{CS}_{2}$ in laser-system-related materials. IEEE. J. Quantum Electron. QE-11, 259-263 (1975).

24 Shishido, A. Rewritable holograms based on azobenzene-containing liquid-crystalline polymers. Polym. J. 42, 525-533 (2010).

25 Hrozhyk, U. A., Serak, S. V., Tabiryan, N. V., Hoke, L., Steeves, D. M. \& Kimball, B. R. Azobenzene liquid crystalline materials for efficient optical switching with pulsed and/or continuous wave laser beams. Opt. Express 18, 8697-8704 (2010).

26 Moreno, I., Davis, J. A., Hernandez, T. M., Cottrell, D. M. \& Sand, D. Complete polarization control of light from a liquid crystal spatial light modulator. Opt. Express 20, 364-376 (2012).

27 Jánossy, I. \& Lloyd, A. D. Low-power optical reorientation in dyed nematics. Mol. Cryst. Liq. Cryst. 203, 77-84 (1991).

28 Zhang, H., Shiino, S., Shishido, A., Kanazawa, A., Tsutsumi, O., Shiono, T. \& Ikeda, T. A thiophene liquid crystal as a novel $\pi$-conjugated dye for photo-manipulation of molecular alignment. Adv. Mater. 12, 1336-1339 (2000).

29 Yaegashi, M., Shishido, A., Shiono, T. \& Ikeda, T. Effect of ester moieties in dye structures on photoinduced reorientation of dye-doped liquid crystals. Chem. Mater. 17, 4304-4309 (2005).

30 Lucchetti, L., Fabrizio, M. D., Francescangeli, O. \& Simoni, F. Colossal optical nonlinearity in dye doped liquid crystals. Opt. Commun. 233, 417-424 (2004). 
31 Bryan-Brown, G. P., Wood, E. L. \& Sage, I. C. Weak surface anchoring of liquid crystals. Nature 399, 338-340 (1999).

32 Liao, Y.-J., Hsu, C.-S. \& Wu, S.-T. Dipping and photo-induced liquid crystal alignments using silane surfactants. Jpn. J. Appl. Phys. 39, L90-L93 (2000).

33 Seki, T., Fukuda, R., Tamaki, T. \& Ichimura, K. Alignment photoregulation of liquid crystals on precisely area controlled azobenzene Langmuir-Blodgett monolayers. Thin Solid Films 243, 675-678 (1994).

34 Aoki, K., Seki, T., Suziki, Y., Tamaki, T., Hosoki, A. \& Ichimura, K. Factors affecting photoinduced alignment regulation of cyclohexanecarboxylate-type nematic liquid crystals by azobenzene molecular films. Langmuir 8, 1007-1013 (1992).

35 Aihara, Y., Kinoshita, M., Wang, J., Mamiya, J., Priimagi, A. \& Shishido, A. Polymer stabilization enhances the orientational optical nonlinearity of oligothiophene-doped nematic liquid crystals. Adv. Opt. Mater. 1, 787-791 (2013)

36 Wang, J., Aihara, Y., Kinoshita, M., Mamiya, J., Priimagi, A. \& Shishido, A. Laserpointer-induced self-focusing effect in hybrid-aligned dye-doped liquid crystals. Sci. Rep. 5, 9890 (2015).

37 Kahn, F. J. Orientation of liquid crystals by surface coupling agents. Appl. Phys. Lett. 22, 386-388 (1973).

38 Shibaev, V. P., Kostromin, S. G. \& Plate, N. A. Thermotropic liquid-crystalline polymers-VI: Comb-like liquid-crystalline polymers of the smectic and nematic types with cyanobiphenyl groups in the side-chains. Eur. Polym. J. 18, 651-659 (1982).

39 Durbin, S. D., Arakelian, S. M. \& Shen, Y. R. Laser-induced diffraction rings from a nematic-liquid-crystal film. Opt. Lett. 6, 411-413 (1981). 